International Journal of Pure and Applied Mathematics

Volume 106 No. 4 2016, 1017-1028

ISSN: 1311-8080 (printed version); ISSN: 1314-3395 (on-line version)

url: http://www.ijpam.eu

doi: $10.12732 /$ ijpam.v106i4.4

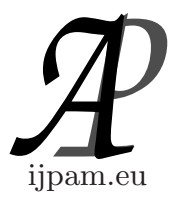

\title{
ON SD-PRIME CORDIAL GRAPHS
}

\author{
Gee-Choon Lau ${ }^{1}$, Hong-Heng $\mathrm{Chu}^{2}$, Nurulzulaiha Suhadak ${ }^{3}$, \\ Fong-Yeng Foo ${ }^{4}$, Ho-Kuen $\mathrm{Ng}^{5}$ \\ ${ }^{1,2,3,4}$ Faculty of Computer \& Mathematical Sciences \\ Universiti Teknologi MARA (Segamat Campus) \\ 85000, Johor, MALAYSIA \\ ${ }^{5}$ Department of Mathematics \\ San Jose State University \\ San Jose, CA 95192, USA
}

\begin{abstract}
Let $G=(V(G), E(G))$ be a simple, finite and undirected graph of order $n$. Given a bijection $f: V(G) \rightarrow\{1, \ldots, n\}$, we associate 2 integers $S=f(u)+f(v)$ and $D=|f(u)-f(v)|$ with every edge $u v$ in $E(G)$. The labeling $f$ induces an edge labeling $f^{\prime}: E(G) \rightarrow\{0,1\}$ such that for any edge $u v$ in $E(G), f^{\prime}(u v)=1$ if $\operatorname{gcd}(S, D)=1$, and $f^{\prime}(u v)=0$ otherwise. Let $e_{f},(i)$ be the number of edges labeled with $i \in\{0,1\}$. We say $f$ is an SD-prime cordial labeling if $\left|e_{f},(0)-e_{f},(1)\right| \leq 1$. Moreover $G$ is SD-prime cordial if it admits an SD-prime cordial labeling. In this paper, we investigate the SD-prime cordiality of some standard graphs.
\end{abstract}

AMS Subject Classification: 05C78, 05C25

Key Words: prime labeling, prime cordial labeling, SD-prime labeling, SD-prime cordial labeling

\section{Introduction}

Let $G=(V(G), E(G))$ (or $G=(V, E)$ for short if not ambiguous) be a simple, finite and undirected graph of order $|V|=n$ and size $|E|=m$. All notation not defined in this paper can be found in [1].

Received: November 22, 2015

Published: March 6, 2016
(C) 2016 Academic Publications, Ltd.

url: www.acadpubl.eu 
In 1967, Rosa introduced the first paper on graph labeling. Since then, there have been more than 1500 research papers on graph labeling (see the dynamic survey by Gallian [5]).

In $[10,11]$, the authors introduced the concept of prime graphs and prime cordial graphs.

Definition 1.1. A bijection $f: V \rightarrow\{1, \ldots, n\}$ induces an edge labeling $f^{\prime}: E \rightarrow\{0,1\}$ such that for any edge $u v$ in $G, f^{\prime}(u v)=1$ if $g c d(f(u), f(v))=1$, and $f^{\prime}(u v)=0$ otherwise. Such a labeling is called a prime labeling if $f^{\prime}(u v)=1$ for all $u v \in E$. We say $G$ is a prime graph if it admits a prime labeling.

For an edge labeling $f^{\prime}: E \rightarrow\{0,1\}$ of a graph $G$, we let $e_{f^{\prime}}(i)$ be the number of edges labeled with $i \in\{0,1\}$.

Definition 1.2. A bijection $f: V \rightarrow\{1,2,3, \ldots, n\}$ induces an edge labeling $f^{\prime}: E \rightarrow\{0,1\}$ such that for any edge $u v$ in $G, f^{\prime}(u v)=1$ if $\operatorname{gcd}(f(u), f(v))=1$, and $f^{\prime}(u v)=0$ otherwise. We say that $f$ is a prime cordial labeling if $\left|e_{f^{\prime}}(1)-e_{f^{\prime}}(0)\right| \leq 1$. Moreover, $G$ is prime cordial if it admits a prime cordial labeling.

Several results on prime and prime cordial graphs can be found in $[2,3,4$, $8,9]$. In [6], Lau and Shiu introduced a variant of prime graph labeling which is defined as follows.

Given a bijection $f: V \rightarrow\{1, \ldots, n\}$, we associate 2 integers $S=f(u)+f(v)$ and $D=|f(u)-f(v)|$ with every edge $u v$ in $E$.

Definition 1.3. A bijection $f: V \rightarrow\{1, \ldots, n\}$ induces an edge labeling $f^{\prime}: E \rightarrow\{0,1\}$ such that for any edge $u v$ in $G, f^{\prime}(u v)=1$ if $\operatorname{gcd}(S, D)=1$, and $f^{\prime}(u v)=0$ otherwise. We say $f$ is an $S D$-prime labeling if $f^{\prime}(u v)=1$ for all $u v \in E$. Moreover, $G$ is SD-prime if it admits an SD-prime labeling.

Lau and Shiu then proved the following necessary and sufficient condition for the existence of an SD-prime labeling.

Theorem 1.1. A graph $G$ of order $n$ is $S D$-prime if and only if $G$ is bipartite and there exists a labeling $f: V \rightarrow\{1,2, \ldots, n\}$ such that for each edge $u v$ of $G, f(u)$ and $f(v)$ are of different parity and $\operatorname{gcd}(f(u), f(v))=1$.

In this paper, we introduce the concept of an SD-prime cordial labeling defined as follows.

Definition 1.4. A bijection $f: V \rightarrow\{1, \ldots, n\}$ induces an edge labeling $f^{\prime}: E \rightarrow\{0,1\}$ such that for any edge $u v$ in $G, f^{\prime}(u v)=1$ if $\operatorname{gcd}(S, D)=1$, and $f^{\prime}(u v)=0$ otherwise. The labeling $f$ is called an SD-prime cordial labeling if $\left|e_{f^{\prime}}(0)-e_{f^{\prime}}(1)\right| \leq 1$. We say that $G$ is $S D$-prime cordial if it admits an 
SD-prime cordial labeling.

We shall drop the subscript $f^{\prime}$ if the context is clear.

\section{Main Results}

In [7], the authors show that every bipartite graph is an induced subgraph of an SD-prime graph. Similarly, we have

Theorem 2.1. Every (complete) bipartite graph $G$ is an induced subgraph of an SD-prime cordial graph.

Proof. Let the bipartition of $G$ be $(A, B)$ such that $|A|=r$ and $|B|=s$. Label the vertices in $A$ by integers in $X=\{1,3, \ldots, 2 r-1\}$, and the vertices in $B$ by integers in $Y=\left\{2^{1}, 2^{2}, \ldots, 2^{s}\right\}$. Now, all the edges of $G$ are labeled 1 . Let $v \geq \max \left\{2 r-1,2^{s}\right\}$ be sufficiently large. We add $v-r-s>0$ new vertices and label them using the integers in $\{1,2, \ldots, v\} \backslash(X \cup Y)$. We can now obtain the required 0 -edges by joining new vertices with odd labels to vertices in $A$ or joining new vertices with even labels to vertices in $B$.

Lemma 2.2. Let $G$ be a graph of size $m$. Consider the conditions (i) $f(u)$ and $f(v)$ are of distinct parity and that $\operatorname{gcd}(f(u), f(v))=1$; and (ii) $f(u)$ and $f(v)$ are of same parity. If, for each possible labeling $f$, the number of $(f(u), f(v))$ pairs that meets one of the conditions is greater than $\left\lceil\frac{m}{2}\right\rceil$, or is less than $\left\lfloor\frac{m}{2}\right\rfloor$, then $G$ is not $S D$-prime.

Proof. A labeling $f$ that meets condition (i) will have too many or too few 1-edges while one that meets condition (ii) will have too many or too few 0-edges. Hence, $f$ is not an SD-prime cordial labeling.

Theorem 2.3. The complete graph $K_{n}$ is SD-prime cordial if and only if $n=2,3,6,7,9,10,11$.

Proof. Let $f: V \rightarrow\{1, \ldots, n\}$ be a bijective labeling of $K_{n}$. It is easy to verify that for $2 \leq n \leq 15, K_{n}$ is SD-prime cordial if and only if $n=$ $2,3,6,7,9,10,11$. Assume $n \geq 16$. We consider 4 cases.

Case (1). $n=4 k, k \geq 4$. In this case, $K_{n}$ has even size $2 k(4 k-1)$. By definition and Theorem 1.1, the number of 1-edges in $K_{n}$ is at most $(2 k)^{2}-$ $\left\lfloor\frac{4 k}{3}\right\rfloor<k(4 k-1)=\frac{e\left(K_{\mathrm{n}}\right)}{2}$. By Lemma $2.2, K_{n}$ is not SD-prime cordial. 
Case (2). $n=4 k+1, k \geq 4$. In this case, $K_{n}$ has even size $2 k(4 k+1)$. By definition and Theorem 1.1, the number of 1-edges in $K_{n}$ is at most $(2 k)(2 k+$ $1)-\left\lfloor\frac{4 k+1}{3}\right\rfloor<k(4 k+1)=\left\lfloor\frac{e\left(K_{\mathrm{n}}\right)}{2}\right\rfloor$. By Lemma 2.2, $K_{n}$ is not SD-prime cordial.

Case (3). $n=4 k+2, k \geq 4$. In this case, $K_{n}$ has odd size $(2 k+1)(4 k+1)$. By definition and Theorem 1.1, the number of 1-edges in $K_{n}$ is at most $(2 k+$ $1)^{2}-\left\lfloor\frac{4 k+2}{3}\right\rfloor<4 k^{2}+3 k=\left\lfloor\frac{e\left(K_{\mathrm{n}}\right)}{2}\right\rfloor$. By Lemma 2.2, $K_{n}$ is not SD-prime cordial.

Case (4). $n=4 k+3, k \geq 4$. In this case, $K_{n}$ has odd size $(2 k+1)(4 k+$ 3). By definition and Theorem 1.1, the number of 1-edges in $K_{n}$ is at most $(2 k+1)(2 k+2)-\left\lfloor\frac{4 k+3}{3}\right\rfloor<4 k^{2}+5 k+1=\left\lfloor\frac{e\left(K_{\mathrm{n}}\right)}{2}\right\rfloor$. By Lemma $2.2, K_{n}$ is not SD-prime cordial.

Given integers $n \geq m \geq 1$. Let $I_{1}=\{1, p:(m+n) / 2<p \leq m+n$ is an odd prime $\}$ and $I_{2}=\left\{2^{a}: 2 \leq 2^{a} \leq m+n, a \geq 1\right\}$.

Theorem 2.4. The complete bipartite graph $K_{m, n}, n \geq m \geq 1$ is $S D$ prime cordial if $\left|I_{1}\right| \geq\left\lceil\frac{m}{2}\right\rceil,\left|I_{2}\right| \geq\left\lfloor\frac{m}{2}\right\rfloor$.

Proof. Let the 2 partite sets of $K_{m, n}$ be $A=\left\{u_{i}, 1 \leq i \leq m\right\}$ and $B=$ $\left\{v_{i}, 1 \leq i \leq n\right\}$. Label the vertices in $A$ by $\left\lceil\frac{m}{2}\right\rceil$ integers in $I_{1}$ and $\left\lfloor\frac{m}{2}\right\rfloor$ integers in $I_{2}$. Label the vertices in $B$ by the remaining integers in $\{1,2, \ldots, m+n\}$. Clearly, $f$ is an SD-prime cordial labeling.

Let $S t(n) \cong K_{1, n}$ denote the star graph.

Corollary 2.5. All star graphs $S t(n)(n \geq 1)$ are SD-prime cordial.

Let $D S(a, b)(b \geq a \geq 2)$ denote the double star graph obtained from $\operatorname{St}(a)$ and $S t(b)$ by adding an edge joining the central vertices of the two star graphs.

Theorem 2.6. All double stars $D S(a, b)$ are SD-prime cordial.

Proof. Let the vertex sets of $S t(a)$ and $S t(b)$ be $\{u\} \cup\left\{u_{i} \mid 1 \leq i \leq a\right\}$ and $\{v\} \cup\left\{v_{j} \mid 1 \leq j \leq b\right\}$, where $u$ and $v$ are the central vertices, respectively. If $a$ and $b$ are of same parity, define $f(u)=1, f(v)=2, f\left(u_{i}\right)=i+2(1 \leq i \leq a)$, and $f\left(v_{j}\right)=a+2+j(1 \leq j \leq b)$. Suppose $a$ and $b$ are of different parity. Let $p$ be the largest prime $\leq a+b+2$. Define $f(u)=1, f(v)=p$, and label the vertices $u_{1}, u_{2}, \ldots, u_{a}, v_{1}, v_{2}, \ldots, v_{b}$ by the integers in $\{2,3,4, \ldots, \ldots, a+b+2\} \backslash\{p\}$ consecutively. Clearly, $f$ is an SD-prime cordial labeling.

In the next theorem, we show that all paths and cycles are SD-prime cordial. The approach used turns out to be very useful in getting an SD-prime cordial labeling for the results that follow. We use the notations $P_{n}=u_{1} u_{2} \cdots u_{n}$ for 
a path and $C_{n}=u_{1} u_{2} \cdots u_{n} u_{1}$ for a cycle in the following constructions using paths and cycles.

Theorem 2.7. All paths $P_{n}(n \geq 2)$ and cycles $C_{n}(n \geq 3)$ are SD-prime cordial.

Proof. We first consider $P_{n}$ in 2 cases.

Case $(1) \cdot n \equiv 0,1,3(\bmod 4)$. Define

$f\left(u_{i}\right)=i$ if $i \equiv 0,1(\bmod 4) ; f\left(u_{i}\right)=i+1$ if $i \equiv 2(\bmod 4) ;$ and $f\left(u_{i}\right)=i-1$ if $i \equiv 3(\bmod 4)$.

Case $(2) . n \equiv 2(\bmod 4)$. Define $f\left(u_{i}\right)=i$ if $i=n-1, n$, and for $i \leq n-2$, $f$ is defined as in Case (1).

It is clear that the induced edge labels are alternating between 0 and 1 except that for $P_{n}, n \equiv 2(\bmod 4)$, the last two edge labels are 1 . Hence, $|e(1)-e(0)| \leq 1$ and $P_{n}$ is SD-prime cordial.

We now consider $C_{n}$. If $n \equiv 0,1,3(\bmod 4)$, we label the vertices in a way similar to that for $P_{n}$. Suppose $n \equiv 2(\bmod 4)$. For $n=6$, label the vertices by $1,4,5,3,6,2$ consecutively. For $n \geq 10$, first define $f$ as in Case (2) above. Note that the labeling gives $e(1)-e(0)=2$. Now, switch the vertex label of 3 and 7 . It is easy to check that $e(1)=e(0)$ and $C_{n}$ is SD-prime cordial.

Definition 2.1. A fan graph of order $n \geq 3$, denoted $F_{n}$, is a graph obtained from a path $P_{n}$ by joining $u_{1}$ to $u_{i}(3 \leq i \leq n)$.

Theorem 2.8. The fan graph $F_{n}$ is $S D$-prime cordial for all $n \geq 4$.

Proof. By the labeling approach as in the proof of Theorem 2.7, it is clear that $F_{n}$ is SD-prime cordial.

Definition 2.2. A double fan graph of order $n \geq 4$, denoted $D F_{n}$, is a graph obtained from a fan graph $F_{n}$ by deleting edge $u_{1} u_{2}$ and joining $u_{2}$ to $u_{i}$ $(4 \leq i \leq n)$.

Theorem 2.9. The double fan graph $D F_{n}$ is $S D$-prime cordial for all $n \geq 4$.

Proof. Suppose $n \equiv 1,2,3(\bmod 4)$. Define $f\left(u_{i}\right)=i$ for $i=1,2$. For $i \geq 3$, define

$f\left(u_{i}\right)=i+1$ for $i \equiv 0(\bmod 4) ; f\left(u_{i}\right)=i-1$ for $i \equiv 1(\bmod 4) ;$ and $f\left(u_{i}\right)=i$ for $i \equiv 2,3(\bmod 4)$.

For $n \equiv 0(\bmod 4)$, define $f\left(u_{i}\right)$ as above if $i \leq n-1$ and $f\left(u_{n}\right)=n$. Clearly, the labeling is SD-prime cordial. 
Definition 2.3. A wheel graph of order $n \geq 4$, denoted $W_{n}$, is a graph obtained from a fan graph $F_{n}$ by joining the two degree 2 vertices of $F_{n}$ by an edge.

Theorem 2.10. The wheel graph $W_{n}$ is SD-prime cordial if and only if $n \geq 5$.

Proof. Note that $E\left(W_{n}\right)=\left\{u_{1} u_{j}(2 \leq j \leq n), u_{i} u_{i+1}(2 \leq i \leq n-1), u_{2} u_{n}\right\}$. By Theorem 2.3, $W_{4}$ is not SD-prime cordial. Assume $n \geq 5$ in the following 3 cases.

Case $(1) . n \equiv 0(\bmod 4)$. Define $f\left(u_{1}\right)=4, f\left(u_{2}\right)=1, f\left(u_{3}\right)=3, f\left(u_{4}\right)=$ 2 , and for $5 \leq i \leq n$,

$f\left(u_{i}\right)=i$ if $i \equiv 1,2(\bmod 4) ; f\left(u_{i}\right)=i+1$ if $i \equiv 3(\bmod 4) ;$ and $f\left(u_{i}\right)=i-1$ if $i \equiv 0(\bmod 4)$.

Next, switch the vertex label of 3 and 5 .

Case $(2) . n \equiv 1,2(\bmod 4)$. Define $f\left(u_{1}\right)=1$ and for $i \geq 2$,

$f\left(u_{i}\right)=i$ if $i \equiv 2,3(\bmod 4) ; f\left(u_{i}\right)=i+1$ if $i \equiv 0(\bmod 4) ;$ and $f\left(u_{i}\right)=i-1$ if $i \equiv 1(\bmod 4)$.

Case $(3) . n \equiv 3(\bmod 4)$. Define $f\left(u_{1}\right)=2, f\left(u_{2}\right)=1, f\left(u_{n}\right)=n$ and for $3 \leq i \leq n-1$,

$f\left(u_{i}\right)=i$ if $i \equiv 0,3(\bmod 4) ; f\left(u_{i}\right)=i+1$ if $i \equiv 1(\bmod 4) ;$ and $f\left(u_{i}\right)=i-1$ if $i \equiv 2(\bmod 4)$.

It is easy to verify that $f$ is an SD-prime cordial labeling.

Lemma 2.11. The ladder $P_{n} \times P_{2}$ is $S D$-prime cordial, for all $n$.

Proof. Assume each copy of $P_{n}$ is a horizontal path. We first consider the labeling for the four simplest cases.

\begin{tabular}{|c|cc|ccc|cccc|}
\hline$n=1$ & $n=2$ & \multicolumn{3}{|c|}{$n=3$} & \multicolumn{4}{|c|}{$n=4$} \\
\hline 1 & 1 & 3 & 1 & 3 & 5 & 1 & 3 & 5 & 8 \\
\hline 2 & 2 & 4 & 2 & 4 & 6 & 2 & 6 & 4 & 7 \\
\hline
\end{tabular}

At this point, we have shown that $P_{n} \times P_{2}$ is SD-prime cordial, for $n=$ $1,2,3,4$. Furthermore, note that in each of the above labelings, the two rightmost vertices are $2 n-1$ and $2 n$.

We will continue to add vertices and edges to one of the above grids. At any point, assume that the highest vertex label is $k$ (which is necessarily on the rightmost edge and adjacent to the vertex with label $k-1$ ). Consider these two constructions.

Construction $C 1$, to be used when $k \equiv 1(\bmod 3)$ : 


\begin{tabular}{|c|c|c|c|c|}
\hline$k$ & $k+1$ & $k+3$ & $k+5$ & $k+8$ \\
\hline$k-1$ & $k+2$ & $k+4$ & $k+6$ & $k+7$ \\
\hline
\end{tabular}

We have joined the two vertices with labels $k$ and $k+1$, and the two vertices with labels $k-1$ and $k+2$. An additional six 0-edges and six 1-edges have been added.

Construction $C 2$, to be used when $k \neq \equiv 1(\bmod 3)$ :

\begin{tabular}{|c|c|c|c|c|}
\hline$k$ & $k+2$ & $k+4$ & $k+6$ & $k+7$ \\
\hline$k-1$ & $k+1$ & $k+3$ & $k+5$ & $k+8$ \\
\hline
\end{tabular}

We have joined the two vertices with labels $k-1$ and $k+1$, and the two vertices with labels $k$ and $k+2$. An additional six 0 -edges and six 1-edges have been added.

Note that the two highest vertex labels are again on the rightmost edge.

For a general $n$, use one of the four simple cases, according to the value of $n$ (mod 4), and use constructions $C 1$ or $C 2$ until $P_{n} \times P_{2}$ has been attained.

Lemma 2.12. The grid $P_{n} \times P_{3}$ is $S D$-prime cordial, for all $n$.

Proof. Assume each copy of $P_{3}$ is a vertical path. We first consider the two simplest cases.

$n=1$ : Label consecutively with 1,3 and 2 .

$n=2$ : Label the left $P_{3}$ and right $P_{3}$ from top to bottom by $1,3,2$ and 4 , 6,5 respectively.

At this point, we have shown that $P_{n} \times P_{3}$ is SD-prime cordial, for $n=1$ and 2. Furthermore, note that in each of the above labelings, the highest three vertex labels are on the rightmost edge. In the labeling for $n=1$, these vertex labels are $\equiv 1,3$, and $2(\bmod 6)$, with the highest vertex label in the middle. In the labeling for $n=2$, these vertex labels are $\equiv 4,0$, and $5(\bmod 6)$, again with the highest vertex label in the middle.

We will continue to add vertices and edges to one of the above grids. At any point, assume that the highest vertex label is $k$ (which is necessarily in the middle of the rightmost edge, and adjacent to the vertices with labels $k-2$ and $k-1)$. Consider adding six vertices, with the vertex labels shown below, to the right. Join the two vertices with labels $k-2$ and $k+1$, the two vertices with labels $k$ and $k+2$, and the two vertices with labels $k-1$ and $k+3$. Note that the three highest vertex labels are still on the rightmost edge, with the highest vertex label in the middle to get the three rightmost $P_{3}$ with labelings as given:

\begin{tabular}{|c|c|c|}
\hline$k-2$ & $k+1$ & $k+5$ \\
\hline$k$ & $k+2$ & $k+6$ \\
\hline$k-1$ & $k+3$ & $k+4$ \\
\hline
\end{tabular}


For a general $n$, use one of the simplest cases, according to the value of $n$ ( $\bmod 2)$, and repeatedly add the block shown above until $P_{n} \times P_{2}$ has been attained. It can be readily verified that, after each step, an additional five 0edges and five 1-edges have been added. Furthermore, in the case when $n \equiv 1$ $(\bmod 2)$, the rightmost vertices are $\equiv 2,3$, and $1(\bmod 6)$, and in the case when $n \equiv 0(\bmod 2)$, the rightmost vertices are $\equiv 5,0$, and $4(\bmod 6)$.

Lemma 2.13. Let $n \geq m \geq 4$. The grid $P_{n} \times P_{m}$ is SD-prime cordial if $n-m \leq 1$.

Proof. View the $m$ copies of $P_{n}$ as $m$ horizontal paths, with the paths stacked one above another. Label the vertices from left to right, top to bottom. In general, the label of the $j$-th vertex of the $i$-th $P_{n}$ is $(i-1) n+j$. Clearly, horizontally adjacent labels have opposite parity and are relatively prime, while vertically adjacent labels have the same parity. Thus all horizontal edges have label 1 and all vertical edges have label 0. Among the $2 m n-m-n$ edges, $m(n-1)$ of them are horizontal and $n(m-1)$ of them are vertical. Thus there are $(n-m)$ more 1-edges than 0-edges. Since $|n-m| \leq 1$, the proof is complete.

Lemma 2.14. For $n \equiv 0(\bmod 4)$ and $n \geq m+2 \geq 6$, the grid $P_{n} \times P_{m}$ is $S D$-prime cordial if $n-1$ and $n+1$ are prime.

Proof. Begin with the vertex labeling as in Lemma 2.13. Since $e(1)-e(0)=$ $n-m \geq 2$, we know the labeling is non-SD-prime cordial. Divide each copy of $P_{n}$ into blocks of four vertices, and consider the following modifications to the vertex labeling.

Modification $M 1$. Changing the top three copies of $P_{n}$ to get:

\begin{tabular}{|c|c|c|c|}
\hline 2 & 1 & 3 & 4 \\
\hline$n+2$ & $n+1$ & $n+3$ & $n+4$ \\
\hline $2 n+2$ & $2 n+1$ & $2 n+3$ & $2 n+4$ \\
\hline $3 n+1$ & $3 n+2$ & $3 n+3$ & $3 n+4$ \\
\hline
\end{tabular}

Each of the top three copies of $P_{n}$ has exactly one 0-edge. We claim that the two leftmost edges joining the third and fourth copies of $P_{n}$ have label 1. Consider the leftmost edge with end-vertex labels $2 n+2$ and $3 n+1$, and assume that there is a prime $p(\geq 3)$ dividing these two integers. Since their difference is $n-1$, which is prime, so $p=n-1$. It is obvious that $(n-1)$ does not divide $(2 n+2)$, a contradiction. Consider the second leftmost edge with end-vertex labels $2 n+1$ and $3 n+2$, and assume that there is a prime $p(\geq 3)$ dividing these two integers. Since their difference is $n+1$, which is prime, so $p=n+1$. 
It is obvious that $(n+1)$ does not divide $(2 n+1)$, a contradiction. All the other edge labels are unchanged. Thus, upon applying modification $M 1$, there are $(n-m-2)$ more 1-edges than 0-edges.

Modification $M 2$. Changing the top two copies of $P_{n}$ to get:

\begin{tabular}{|c|c|c|c|}
\hline 1 & 3 & 2 & 4 \\
\hline$n+1$ & $n+3$ & $n+2$ & $n+4$ \\
\hline $2 n+1$ & $2 n+2$ & $2 n+3$ & $2 n+4$ \\
\hline
\end{tabular}

Each of the top two copies of $P_{n}$ has exactly two 0-edges. We claim that the second and third edges (from the left) joining the second and third copies of $P_{n}$ have label 1. Consider the second edge with end-vertex labels $n+3$ and $2 n+2$, and assume that there is a prime $p(\geq 3)$ dividing the two vertically adjacent vertices. Since their difference is $n-1$, which is prime, so $p=n-1$. It is obvious that $(n-1)$ does not divide $(n+3)$, a contradiction. Consider the third edge with end-vertex labels $n+2$ and $2 n+3$, and assume that there is a prime $p(\geq 3)$ dividing the two vertically adjacent vertices. Since their difference is $n+1$, which is prime, so $p=n+1$. It is obvious that $(n+1)$ does not divide $(n+2)$, a contradiction. All the other edge labels are unchanged. Thus, upon applying modification $M 2$, there are $(n-m-4)$ more 1-edges than 0-edges.

Modification $M 3_{i}, 1 \leq i \leq(n / 4)-1$. Changing the top two copies of $P_{n}$ to get:

\begin{tabular}{|c|c|c|c|}
\hline $4 i+1$ & $4 i+3$ & $4 i+2$ & $4 i+4$ \\
\hline$n+4 i+1$ & $n+4 i+3$ & $n+4 i+2$ & $n+4 i+4$ \\
\hline $2 n+4 i+1$ & $2 n+4 i+2$ & $2 n+4 i+3$ & $2 n+4 i+4$ \\
\hline
\end{tabular}

The argument is similar to that in modification $M 2$. Thus, upon applying modification $M 3_{i}$, the difference $e(1)-(0)$ is reduced by 4 .

For $m \equiv 2,3(\bmod 4)$, combining modification $M 1$ and modifications $M 3_{i}$ incrementally, the difference $e(1)-e(0)$ has values $n-m-2, n-m-6, n-$ $m-10, \ldots,-m+2$.

For $m \equiv 0,1(\bmod 4)$, combining modification $M 2$ and modifications $M 3_{i}$ incrementally, the difference $e(1)-e(0)$ has values $n-m-4, n-m-8, \ldots,-m$.

Thus the difference $|e(1)-e(0)|$ must attain 0 or 1 at some point.

Lemma 2.15. For $n \equiv 0(\bmod 4)$ and $n \geq m+2 \geq 6$, the grid $P_{n+1} \times P_{m}$ is $S D$-prime cordial if $n-1$ and $n+1$ are prime.

Proof. Label the vertices of the subgrid $P_{n} \times P_{m}$ with the (original) vertex labeling as in Lemma 2.13. Label the vertices of the rightmost unlabeled path 
$P_{m}$ by $m n+1$ to $m n+m$ from top to bottom. Observe that all the $(m-1)$ rightmost vertical edges must get the label 1 and the number of rightmost horizontal edges that get the label 0 must be an integer in $[m / 2+1, m]$ for even $m$; or in $[(m+1) / 2, m]$ for odd $m$.

If $m$ is even, $e(1)-e(0)$ must be an integer in $\{n-3, n-5, \ldots, n-m-1\}$. Suppose $e(1)-e(0)>1$. By combining modifications $M 1$ and $M 3_{i}$ described in the proof of Lemma 2.14 incrementally, we see that the difference $e(1)-e(0)$ must eventually attain the value \pm 1 since the decrement of $e(1)-e(0)$ must be an integer in $\{2,6,10,14, \ldots, n-2\}$. If $m$ is odd, $e(1)-e(0)$ must be an integer in $\{n-2, n-4, n-6, \ldots, n-m-1\}$. Suppose $e(1)-e(0)>1$. If $e(1)-e(0) \equiv 0(\bmod 4)$, we use modifications $M 2$ and $M 3_{i}$ described in the proof of Lemma 2.14 incrementally. The difference $e(1)-e(0)$ must eventually attain the value 0 since the decrement of $e(1)-e(0)$ must be an integer in $\{4,8,12, \ldots, n\}$. If $e(1)-e(0) \equiv 2(\bmod 4)$, we use modifications $M 1$ and $M 3_{i}$ incrementally. The difference $e(1)-e(0)$ must eventually attain 0 since the decrement of $e(1)-e(0)$ must be an integer in $\{2,6,10, \ldots, n-2\}$.

Lemma 2.16. For $n \equiv 0(\bmod 4)$ and $n \geq m+2 \geq 6$, the grid $P_{n+2} \times P_{m}$ is $S D$-prime cordial if $n-1$ and $n+1$ are prime.

Proof. Label the vertices of the subgrid $P_{n} \times P_{m}$ with the original vertex labeling as in Lemma 2.13.

We first consider an even $m$. For the two rightmost unlabeled paths $P_{m}$, we label the vertices of the left path $P_{m}$ by $m n+1$ to $m n+m$ from top to bottom, and label the vertices of the right path $P_{m}$ by $m n+m+1$ to $m n+2 m$ from top to bottom. Observe that all the $(m-1)$ rightmost vertical edges must get the label 1 and all the $m$ rightmost horizontal edges must get the label 0 . Hence, $e(1)-e(0)$ must be an integer in $\{n-4, n-6, \ldots, n-m-2\}$. Suppose $e(1)-e(0)>1$. If $e(1)-e(0) \equiv 0(\bmod 4)$, we use modifications $M 2$ and $M 3_{i}$ as described in the proof of Lemma 2.14 incrementally. The difference $e(1)-e(0)$ must eventually attain the value 0 since the decrement of $e(1)-e(0)$ must be an integer in $\{4,8,12, \ldots, n\}$. If $e(1)-e(0) \equiv 2(\bmod 4)$, we use modifications $M 1$ and $M 3_{i}$ incrementally. The difference $e(1)-e(0)$ must eventually attain 0 since the decrement of $e(1)-e(0)$ must be an integer in $\{2,6,10, \ldots, n-2\}$.

Now consider an odd $m$. For the two rightmost unlabeled paths $P_{m}$, we label the vertices of the left path $P_{m}$ by $m n+1, m n+2, \ldots, m n+m-1, m n+2 m$, and label the vertices of the right path $P_{m}$ by $m n+m, m n+m+1, m n+m+$ $2, \ldots, m n+2 m-1$ from top to bottom. Observe that $e(1)-e(0)$ must be an integer in $\{n-5, \ldots, n-m-2\}$. Suppose $e(1)-e(0)>1$. By combining modifications $M 1$ and $M 3_{i}$ incrementally, we see that the difference $e(1)-e(0)$ 
must eventually attain the value \pm 1 since the decrement of $e(1)-e(0)$ must be an integer in $\{2,6,10,14, \ldots, n-2\}$.

Lemma 2.17. For $n \equiv 0(\bmod 4)$ and $n \geq m+2 \geq 6$, the grid $P_{n+3} \times P_{m}$ is $S D$-prime cordial if $n-1$ and $n+1$ are prime.

Proof. Label the vertices of the subgrid $P_{n} \times P_{m}$ with the original vertex labeling as in Lemma 2.13.

We first consider an even $m$. For the three rightmost unlabeled paths $P_{m}$, we label the vertices of the left, middle and right subpath $P_{m}$ from top to bottom respectively by $m n+1$ to $m n+m, m n+m+1$ to $m n+2 m$, and $m n+2 m+1$ to $m n+3 m$. Observe that $e(1)-e(0)$ must be an integer in $\{n-5, n-7, \ldots, n-m-3\}$. Suppose $e(1)-e(0)>1$. By combining modifications $M 1$ and $M 3_{i}$ as described in the proof of Lemma 2.14 incrementally, we see that the difference $e(1)-e(0)$ must eventually attain the value \pm 1 since the decrement of $e(1)-e(0)$ must be an integer in $\{2,6,10,14, \ldots, n-2\}$.

Now consider an odd $m$. For the three rightmost unlabeled paths $P_{m}$, we label the vertices of the left, middle and right subpath $P_{m}$ from top to bottom respectively by $m n+1, m n+2, \ldots, m n+m-1, m n+3 m$, by $m n+$ $m, m n+m+1, \ldots, m n+2 m-2, m n+3 m-1$, and by $m n+2 m-1, m n+$ $2 m, \ldots, m n+3 m-3, m n+3 m-2$. Observe that $e(1)-e(0)$ must be an integer in $\{n, n-2, \ldots, n-m-3\}$. Suppose $e(1)-e(0)>1$. If $e(1)-e(0) \equiv 0(\bmod 4)$, we use modifications $M 2$ and $M 3_{i}$ as described in the proof of Lemma 2.14 incrementally. The difference $e(1)-e(0)$ must eventually attain the value 0 since the decrement of $e(1)-e(0)$ must be an integer in $\{4,8,12, \ldots, n\}$. If $e(1)-e(0) \equiv 2(\bmod 4)$, we use modifications $M 1$ and $M 3_{i}$ incrementally. The difference $e(1)-e(0)$ must eventually attain 0 since the decrement of $e(1)-e(0)$ must be an integer in $\{2,6,10, \ldots, n-2\}$.

We have in Lemmas 2.14 to 2.17 shown that $P_{n+k} \times P_{m}$ is SD-prime cordial for $k=0,1,2,3$ and $n \geq m+2 \geq 6$ if $n \equiv 0(\bmod 4)$ and $n-1, n+1$ are primes. We end the paper with the following conjecture:

Conjecture 2.1. The grid $P_{n} \times P_{m}$ is $S D$-prime cordial for all $n \geq m \geq 2$. 


\section{Aknowledgements}

Research supported by UiTM Johor Research Grant No. 600-UiTMCJ(PJIA. $5 / 2)$.

\section{References}

[1] J.A. Bondy, U.S.R. Murty, Graph theory with applications, New York, MacMillan, 1976.

[2] J. Baskar Babujee and L. Shobana, Prime cordial labelings, Int. Review on Pure and Appl. Math., 5 (2009) 277-282.

[3] J. Baskar Babujee and L. Shobana, Prime and prime cordial labeling for some special graphs, Int. J. Contemp. Math. Sciences, 5 (2010) 2347-2356.

[4] H.L. Fu and K.C. Huang, On prime labeling, Discrete Math., 127 (1994) 181-186.

[5] J.A. Gallian, A dynamic survey of graph labeling, Electronic J. Comb., 19 (2012) \#DS6.

[6] G.C. Lau and W.C. Shiu, On SD-prime labeling of graphs, Utilitas Math., accepted.

[7] G.C. Lau, W.C. Shiu, H.K. Ng, C.D. Ng and P. Jeyanthi, Further results on SD-prime labeling, $J C M C C$, accepted.

[8] M.A. Seoud and M.A. Salim, Two upper bounds of prime cordial graphs, JCMCC, 75 (2010) 95-103.

[9] M.A. Seoud and M.Z. Youssef, On prime labelings of graphs, Congr. Numer., 141 (1999) 203-215.

[10] M. Sundaram, R. Ponraj and S. Somasundram, Prime Cordial Labeling of Graphs, J. Ind. Acad. of Maths., 27(2) (2005), 373-390.

[11] A. Tout, A.N. Dabboucy, and K. Howalla, Prime labeling of graphs, Nat. Acad. Sci. Letters, 11 (1982) 365-368. 